Radial and Nonradial Pulsations as Probes of Stellar Physics

ASP Conference Series, Vol. 259, 2002

C. Aerts, T.R. Bedding, \& J. Christensen-Dalsgaard, eds.

\title{
AGAPEROS Variables in the LMC
}

\author{
M. Schultheis ${ }^{1}$
}

Institut d'Astrophysique de Paris, France

T. Lebzelter ${ }^{1}$

Institut für Astronomie, Vienna, Austria

A. L. Melchior

Observatoire de Paris, DEMIRM, Paris, France

\begin{abstract}
In this work, we present preliminary results based on an LMC Bar sample (AGAPEROS) of $\sim 400$ variable stars combined with near-Infared photometry (DENIS). About $50 \%$ of our objects are semiregular Variables (SRVs) while $20 \%$ are regular Variables with a period range from $30^{\mathrm{d}}$ up to $600^{\mathrm{d}}$. A second period is present in $\sim 40 \%$ of the SRVs. We find a large fraction of regular variables with $P<100^{d}$ which could be SRVs undergoing a phase of periodic variability. The Period-Colour relation $(\log P$ versus $I-J)$, unstudied so far on the LMC, differs from the one found in the Galactic bulge: an effect of metallicity?
\end{abstract}

\section{Introduction}

The late stages of stellar evolution are characterised by regular and irregular variability, a well-known signature of the stellar pulsation of AGB stars. We present a preliminary analysis of AGAPEROS variables (Melchior et al., 2000) - based on the EROS-1 data set, initially motivated by microlensing searches (see Arnaud et al., 1994, Melchior et al., 1999).

\section{AGAPEROS data}

We have studied the AGAPEROS catalogue of 393 variable stars (out of 632 objects) detected over a $0.25 \mathrm{deg}^{2}$ field in the LMC Bar (Melchior et al., 2000). These stars have been selected on the basis of their variability during a 120 day window, with a bias towards long-timescale variations ( $>$ few days), which are not necessarily periodic. See Melchior et al. (2000) for a more detailed description of the data treatment.

\footnotetext{
${ }^{1} \mathrm{MS}$ is supported by the Fonds zur Förderung der wissenschaftlichen Forschung (FWF), Austria, under the project number J1971-PHY. TL has been supported by the Austrian Science Fund under project number P14365-PHY.
} 
To derive the periods, a Fourier analysis of the light curves has been combined with visual inspection to deal also with semiregular and irregular light changes (Lebzelter, 1999). We identified the major period(s) to roughly resemble the overall light change. From the visual inspection we classified the light curves on the regularity and type of their light change into four groups: Regular Variables, semiregular Variables, irregular Variables and other Variables, which include a number of possible binaries.

Note that this classification does not take into account the amplitude of the variation as in the GCVS classification. If more than one period is detected we haven chosen a 'primary' period based on its amplitude and its contribution in the light change. However, this decision is somewhat subjective when the amplitudes are similar.

\section{Results and Discussion}

About $50 \%$ of our sample are semiregular Variables, while $20 \%$ are regular variables with a period range from $30^{\mathrm{d}}$ up to $600^{\mathrm{d}}$. A second period is present in $\sim 40 \%$ of the SRVS. Fie find a large number of regular variables with $\mathrm{P}<100^{\mathrm{d}}$ which could be semiregular Variables undergoing a phase of periodic variability. The period distribution of both regular and semiregular Variables show the maximum at the shortest periods $\left(\mathrm{P}<100^{\mathrm{d}}\right)$.

$80 \%$ of the AGAPEROS Variables show a DENIS counterpart (IJK). We find for the AGAPEROS Variables similar PK-relations as Wood (2000) from MACHO data

The AGAPEROS Variables follow a tight $\log P$ versus $I-J$ relation. Relying on MACHO data in the Galactic Bulge, Schultheis \& Glass (2001) demonstrated that semiregular Variables in the Galactic Bulge show a noticeable scatter in $I-J$ (3-4 mag) along the $\log P$ vs $I-J$ relation. The most significant difference between the Galactic Bulge and the LMC is the smaller range in $I-J$ for the LMC $\left(\sim 1<(I-J)_{0}<2 \mathrm{mag}\right)$ than for the Bulge $\left(\sim 1<(I-J)_{0}<4 \mathrm{mag}\right)$.

The large scatter and the wide $I-J$ range in the Bulge sample compared to the LMC might be explained by the difference in metallicity between the galactic Bulge and the LMC.

A more detailed quantitative analysis using realistic model atmospheres of AGB stars (including metallic lines) is necessary, to fully understand this systematic difference in the $I-J$ colour between the galactic Bulge and the LMC.

\section{References}

Arnaud, M., et al. 1994, Exp. Astron., 4, 265

Lebzelter, T. 1999, A\&A, 351, 644

Melchior, A.L., et al. 1999, A\&AS, 134, 377

Melchior, A.L., Hughes, S.M.G., \& Guibert, J. 2000, A\&AS, 145, 11

Schultheis, M.\& Glass, I.S. 2001, MNRAS, in press

Wood, P.R. 2000, PASA, 17, 18 